\title{
Prevenção de drogas em contextos educacionais: uma análise da rede de colaboração entre pesquisadores
}

\author{
Drug prevention in educational contexts: an analysis of the collaboration \\ network of researchers
}

\section{Prevención de drogas en contextos educacionales: un análisis de la red de colaboración entre investigadores}

\author{
Julia Ferreira Bernardo ${ }^{1, a}$ \\ juliaf.bernardo@hotmail.com | https://orcid.org/0000-0002-4111-4069 \\ Jesús Pascual Mena-Chalco ${ }^{2, b}$ \\ jesus.mena@ufabc.edu.br | https://orcid.org/oooo-0001-7509-55.32 \\ Denise De Micheli, ${ }^{i, c}$ \\ demicheli.unifesp@gmail.com | https://orcid.org/0000-0001-8546-4354
}

\footnotetext{
${ }^{1}$ Universidade Federal de São Paulo. São Paulo, SP, Brasil.

2 Universidade Federal do ABC. Santo André, SP, Brasil.

a Mestrado em Educação pela Universidade Estadual Paulista.

b Doutorado em Ciência da Computação pela Universidade de São Paulo.

c Doutorado em Psicobiologia pela Universidade Federal de São Paulo.
}

\section{Resumo}

O conhecimento da rede de pesquisadores que atuam na área de prevenção de drogas em contextos educacionais é uma forma possível de integralizar e compartilhar informações sobre o tema. O objetivo do estudo aqui apresentado é identificar esses pesquisadores, bem como suas produções acadêmicas, localização geográfica e participação nessa rede de colaboração científica. Para isto, realizou-se um estudo exploratório e descritivo com abordagem quantitativa. Identificaram-se 143 pesquisadores que publicam sobre o tema, sendo, em sua maioria, pesquisadores da área da saúde que colaboram com um ou mais membros da rede. Foram obtidas 154 publicações sobre o tema. A análise da rede evidenciou maior articulação e parcerias entre os pesquisadores da área da saúde, e nenhum relacionamento destes com os pesquisadores da educação. Desta forma, conclui-se que o tema em questão necessita de maiores parcerias e integralidades de informações entre os campos do conhecimento envolvidos, para que a ciência produzida no âmbito acadêmico culmine em ações práticas mais eficazes para a prevenção de drogas em contextos educacionais.

Palavras-chave: Rede social; Pesquisadores; Prevenção; Drogas; Educação. 


\begin{abstract}
The knowledge of the researcher network associated with the study of drugs prevention in educational contexts is a possible way of integrating and sharing information on the subject. The objective of the study presented here is to identify these researchers, their academic productions, geographical location, as well as their participation in this scientific collaboration network. To carry out this task, it was developed a study using an exploratory, descriptive and quantitative approach. We identified 143 researchers, that publish on the subject, mostly associated with the health studies, and collaborate with one or more members of the network. The method allowed to gather 154 publications on the subject. The analysis of the collaboration network showed a more substantial connection and partnership between the health researchers and no relationship between them and the education researchers. Thus the theme examined needs to establish further partnerships and to develop - based on the integrality concept - integrated information between the fields of knowledge involved in the matter so that the science produced in academic level can contribute to provide more effective practical actions aiming the drug prevention in educational contexts.
\end{abstract}

Keywords: Social network; Researchers; Prevention; Drugs; Education.

\title{
Resumen
}

El conocimiento de la red de investigadores que actúan en el área de prevención de drogas en contextos educacionales es una forma posible de integrar y compartir informaciones sobre el tema. El objetivo del estudio presentado aquí es identificar esos investigadores, así como sus producciones académicas, la ubicación geográfica y su participación en esa red de colaboración científica. Para cumplir nuestro objetivo, desarrollamos una investigación exploratoria y descriptiva con abordaje cuantitativo. Encontramos 143 investigadores que publicaron sobre el tema, pertenecientes principalmente al área de la salud, que colaboraron con uno o más miembros de la red. Hemos obtenido 154 publicaciones sobre el tema. El análisis de la red mostró una mayor asociación y colaboración entre los investigadores del área de la salud, y se evidenció que no existe ninguna relación entre ellos y los investigadores del área de la educación. Así, el tema en cuestión necesita mayores colaboraciones entre los investigadores y integraciones (en el sentido del concepto de integralidad) de las informaciones entre los campos del conocimiento involucrados en el asunto para que la ciencia producida en el ámbito académico culmine en acciones prácticas más eficaces para la prevención de drogas en contextos educacionales.

Palabras clave: Red social; Investigadores; Prevención; Drogas; Educación.

Contribuição dos autores: todos os autores contribuíram igualmente na concepção, desenho do estudo, aquisição, análise, interpretação dos dados, redação do artigo e revisão crítica do conteúdo.

Declaração de conflito de interesses: não há.

Fontes de financiamento: Conselho Nacional de Desenvolvimento Científico e Tecnológico (Capes) e Conselho Nacional de Desenvolvimento Científico e Tecnológico (CNPq).

Considerações éticas: pesquisa documental baseada em dados de domínio público e acesso irrestrito. O projeto de doutorado do qual se vincula este estudo foi submetido ao CEP-UNIFESP, via plataforma Brasil, com parecer consubstanciado aprovado $n^{\circ} 2.450 .045$.

Agradecimentos/Contribuições adicionais: não há.

Histórico do artigo: submetido: 17 jul. 2018 | aceito: 05 jun. 2019 | publicado: 12 set. 2019.

Apresentação anterior: não houve.

Licença CC BY-NC atribuição não comercial. Com essa licença é permitido acessar, baixar (download), copiar, imprimir, compartilhar, reutilizar e distribuir os artigos, desde que para uso não comercial e com a citação da fonte, conferindo os devidos créditos de autoria e menção à Reciis. Nesses casos, nenhuma permissão é necessária por parte dos autores ou dos editores. 


\section{Introdução}

Na última década, inúmeros estudos têm evidenciado uma discussão calorosa a respeito da implantação e execução de projetos de prevenção ao uso de drogas no contexto educacional ${ }^{1-3}$. Isso deve-se ao consumo cada vez mais precoce de substâncias entre os adolescentes, bem como ao consenso de que a escola é um lugar privilegiado para se fazer prevenção, uma vez que é neste local que crianças e adolescentes passam grande parte do dia ${ }^{1,4-5}$.

Entretanto, a falta de consenso sobre os aspectos relacionados às drogas, a falta de políticas públicas e integralidade de informações sobre o tema esbarram na clareza quando se pensa em prevenção, além de dificultar o manejo de ações exequíveis nesta área.

Relativamente pouco se conhece sobre o processo de implantação dos projetos de prevenção do uso de drogas no Brasil, tampouco sobre a efetividade destes ${ }^{6}$. Segundo alguns autores, a dificuldade observada de tomar medidas de prevenção e/ou replicar modelos já existentes deve-se à falta de articulação e/ou conhecimento sobre o que, de fato, tem sido feito em matéria de ações preventivas e divulgação de seus resultados $^{7-8}$. De acordo com estudo realizado por pesquisadores do United Nations Office for Drug Control and Crime Prevention (UNODCCP) 9 é fundamental que os programas preventivos ao uso de risco de drogas sejam avaliados e sejam identificadas e disseminadas as ações que se mostrarem eficazes ${ }^{4-5}$.

Nos Estados Unidos, por exemplo, a prevalência de programas de prevenção de drogas em escolas é de $72 \%$, embora sejam discutíveis alguns dos modelos adotados ${ }^{10}$. A Organização Mundial de Saúde (OMS ou, na sigla em inglês, WHO $)^{11}$ considera a prevenção do uso de risco de drogas como um dos temas mais importantes a serem trabalhados, especialmente no que tange à população adolescente, e ressalta a necessidade de maior articulação entre os atores principais desse processo, a saber: cientistas e educadores.

Diante disto, Ferraz et al. ${ }^{12}$ reforçam a necessidade do desenvolvimento de redes articuladas, robustas e sistematizadas que auxiliem na integralização e compartilhamento de informações de forma acessível e confiável, permitindo uma discussão progressiva e adequada com vistas a ações práticas.

Os estudos sobre redes de colaboração têm crescido nas últimas décadas ${ }^{12-15}$ e os benefícios do trabalho colaborativo tem adquirido destaque no cenário científico mundial ${ }^{14}$. Este fato se refere diretamente a uma nova forma de organização social presente em várias esferas da vida. Hoje, a perspectiva de redes, nas quais as pessoas atuam em colaboração, faz mais sentido para tempos onde os contextos coorporativos, acadêmicos, pessoais e/ou outros visam à troca de informações. O trabalho em rede de colaboração transcorre no sentido do desenvolvimento e evolução de conceitos, promoção da ciência e proposição de políticas públicas, e os atores ligados a tais processos se conectam em prol do desenvolvimento social.

Em relação ao campo científico e acadêmico especificamente, Gatti ${ }^{16}$ afirma que nenhum pesquisador trabalha sozinho e a condição essencial para realização de investigações e avanços nos conhecimentos sobre determinado assunto se deve à intercomunicação com pares, trabalho em equipe e parcerias. Atualmente, observa-se certa tendência à realização de trabalhos em colaboração, principalmente em algumas áreas das ciências, seja devido aos fenômenos complexos com os quais os pesquisadores têm se deparado, seja por uma nova lógica social que demanda o estabelecimento de outras e/ou o nascimento de novas relações entre atores ou papéis diferentes desempenhados por eles na colaboração ${ }^{17}$. Na área de saúde, por exemplo, o trabalho em colaboração se apresenta como fundamental, sendo predominante nos veículos de comunicação científica e publicações da área ${ }^{13}$.

Desse modo, considerando a possibilidade de atuação dos vários e diferentes atores e setores na prevenção de drogas em contextos educacionais, o trabalho colaborativo pode ser um dos caminhos para o compartilhamento de informações científicas que desencadeiem em práticas mais efetivas de prevenção.

Em um estudo anterior, realizado por Bernardo e colaboradores ${ }^{18}$, foi descrito um método para mapeamento de pesquisadores precursores, seniores da área em questão, identificados por meio do Catálogo 
de teses e dissertações da Capes, a fim de iniciar a identificação dos pesquisadores da área de prevenção de drogas em contextos educacionais no Brasil. Os resultados mostraram a existência de 61 pesquisadores que orientaram, ao menos, dois trabalhos sobre o tema entre os anos de 1987 e $2011^{18}$.

Considerando as complexas questões envolvendo a prevenção de drogas em contextos educacionais, o crescimento de trabalhos em colaboração na área de saúde, os benefícios proporcionados pela formação e articulação de redes e as oportunidades de estreitamento, por parte dos cientistas, das demandas sociais, o objetivo do estudo aqui apresentado foi identificar a rede de pesquisadores que atua sobe o tema em âmbito nacional, a partir da expansão do mapeamento de pesquisadores precursores ${ }^{18}$. Foram identificados seus descendentes acadêmicos (orientandos de mestrado, doutorado e pós-doutorado) e selecionados aqueles que publicaram na área de interesse, tendo em vista as seguintes questões: a) Quantos são os pesquisadores brasileiros da área em questão?; b) Quais as características deste grupo em relação ao ano de titulação acadêmica e localização geográfica?; c) Como esta rede está configurada e quais são as relações de coautoria existentes entre seus membros?; d) Há diferenças quanto à colaboração por área de atuação? e) Quais são os veículos de comunicação científica utilizados e quantidade de publicação dessa rede?

São muitos os questionamentos ainda a serem feitos e cujas respostas são fundamentais para agregar conhecimento e compartilhá-los sobre essa importante questão. Este trabalho não tem o intuito de esgotar as questões, mas de identificar os principais pesquisadores da área e, quiçá, contribuir para efetivação de redes e compartilhamento de informações.

\section{Método}

Este estudo foi desenvolvido de forma exploratória e descritiva, com a caracterização sistemática das questões pertinentes à pesquisa e análise dos resultados através de abordagem quantitativa.

Para identificação da rede de pesquisadores da área de prevenção de drogas em contextos educacionais e suas colaborações acadêmicas, foram utilizados os dados obtidos e descritos previamente no trabalho de Bernardo e colaboradores ${ }^{18}$, sobre o mapeamento dos pesquisadores precursores. Buscou-se realizar o refinamento de tais dados para identificação de seus descendentes acadêmicos diretos (orientandos de mestrado, doutorado e pós-doutorado) e posterior seleção dos pesquisadores com produção científica na área de interesse. Para tal, foram realizadas quatro etapas apresentadas na Figura 1 e descritas a seguir.

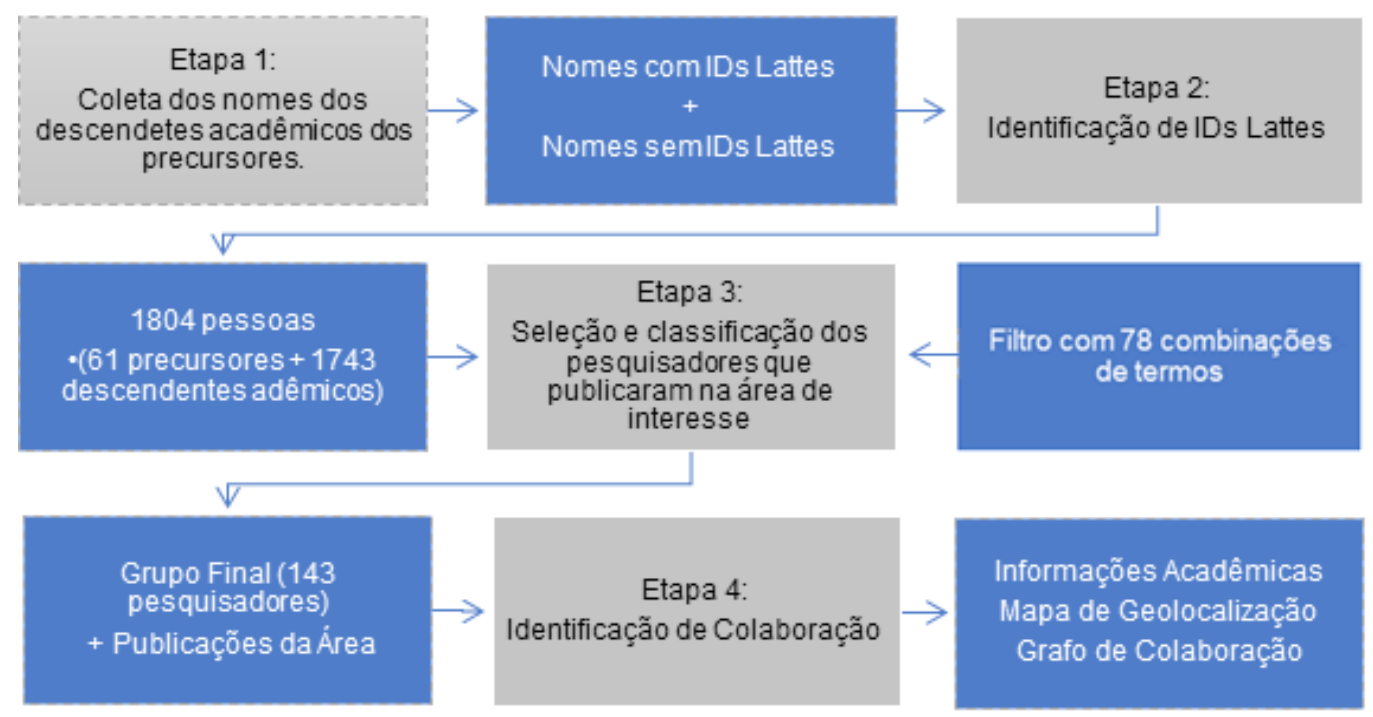

Figura 1 - Fluxograma dos procedimentos metodológicos utilizados para a identificação dos pesquisadores na área de prevenção de drogas em contextos educacionais Fonte: Os autores (2018). 


\section{Etapa 1 - Coleta dos nomes dos descendentes acadêmicos: identificação dos descendentes diretos dos pesquisadores precursores}

A partir da identificação dos 61 pesquisadores precursores da área ${ }^{18}$, foi extraído do currículo Lattes de cada um o nome de seus descendentes acadêmicos na seção 'orientações concluídas'. Ou seja, para cada pesquisador precursor, foi possível listar o nome de todos os seus descendentes diretos (orientandos de mestrado, doutorado e pós-doutorado) em um documento único, Excel. Nessa planilha foram registrados os nomes daqueles já associados diretamente ao currículo dos precursores ${ }^{i}$ com seus respectivos identificadores de currículos Lattes (ID Lattes) ${ }^{\mathrm{ii}}$ e descendentes sem ID Lattes associado. No caso desses descendentes sem associação ao currículo dos precursores, os quais somaram 756 nomes, um procedimento adicional foi realizado, como especificado na etapa 2.

\section{Etapa 2 - Identificação de IDs Lattes de descendentes acadêmicos}

Para identificação dos descendentes sem IDs Lattes associado aos currículos de seus orientadores (precursores), realizou-se uma busca no sistema web disponível na plataforma Lattesiii. O objetivo desta busca era verificar possíveis homônimos, encontrar pessoas que mudaram de nome após casamento ou separação e/ou encontrar sobrenomes completos, que constavam diferentes no currículo do precursor.

Dos 756 nomes sem IDs, 597 foram identificados. No entanto, 159 não foram encontrados com nenhuma estratégia de busca (correspondente a somente $7 \%$ de todos os nomes obtidos). Acredita-se que, tais nomes ou eram de pessoas que não possuíam currículo Lattes ou seus nomes estavam muito diferentes na plataforma Lattes daqueles inseridos no currículo do precursor. Somando os pesquisadores identificados em todas as etapas ( 1 e 2) com os pesquisadores precursores previamente identificados, obteve-se uma lista de 1.804 pessoas.

\section{Etapa 3 - Seleção e classificação dos pesquisadores que publicaram na área de interesse}

Para selecionar somente os pesquisadores que possuíam publicações na área de prevenção de drogas em contextos educacionais, dentre as 1.804 pessoas (61 pesquisadores precursores e 1.743 orientandos de mestrado, doutorado e pós-doutorado), foi aplicado um filtro contendo 78 combinações de termos, utilizados como estratégia de busca, relativos à área de interesse. Para definição dos termos, foi realizada uma pesquisa ampla em trabalhos da área (artigos, teses, dissertações, descritores em ciências da saúde DeCS, entre outros, da base de dados Bireme) que pudessem indicar as palavras mais utilizadas nos títulos dos trabalhos sobre prevenção de drogas em contextos educacionais. Esta seleção de combinações de termos foi realizada anteriormente por Bernardo e colaboradores ${ }^{18}$ e utilizada neste estudo, por considerarmos uma lista completa e bem estruturada de palavras correlatas à área.

Esse filtro com as 78 combinações de termos $^{\text {iv }}$ foi aplicado para selecionar aqueles pesquisadores que continham, ao menos, uma ocorrência das combinações nos títulos de publicações referentes a: resumos simples e expandidos em congressos, livros e capítulos de livros, artigos em periódicos, em seu currículo Lattes. Foram selecionados 143 pesquisadores.

\footnotetext{
i Essa associação direta se refere ao link criado entre currículos, quando aparece a figura (9) e é possível acessar diretamente o currículo da pessoa mencionada.

ii ID Lattes é o número de identificação (composto de 16 dígitos) de cada currículo. Cada ID é único a cada pessoa registrada na Plataforma Lattes.

iii Disponível em: http://buscatextual.cnpq.br/buscatextual/busca.do?metodo=apresentar.

iv Os termos mais recorrentes, encontrados nos currículos dos pesquisadores foram: educacao AND drogas; escola* AND drogas AND prevenção; adolescente* AND drogas AND escola*; educador* AND drogas AND prevenção.
} 
Além disso, essa etapa possibilitou a extração das informações dos respectivos currículos, exportadas para tabelas em um documento Excel com: o nome de cada pesquisador, os dados sobre a estratégia de busca - termos e frequência encontrada em cada currículo e cada publicação, cada tipo de publicação identificada e as informações adjacentes a elas (por exemplo, veículo de publicação, ano de publicação, coautores da publicação).

Para identificar a área principal de atuação de cada pesquisador, realizou-se uma classificação, por meio da avaliação criteriosa do currículo Lattes de cada um deles, observando suas publicações, sua associação a grupos de pesquisa, projetos e resumo (resumo ou autobiografia) geral do currículo. Esta avaliação foi realizada por dois pesquisadores, que puderam definir três áreas principais: saúde, educação e educação e saúde, sendo esta última subdividida em três subáreas: mais saúde do que educação; mais educação do que saúde e multidisciplinar. Esta subdivisão da área de educação e saúde foi necessária para diferenciar pesquisadores que, embora trabalhem com uma interface interdisciplinar, atuam mais intensamente em um ou outro tema (mais educação ou mais saúde), ou mesmo transitam igualmente nas duas áreas (multidisciplinar).

Essas categorias foram consideradas no trabalho de Bernardo e colaboradore ${ }^{18} \mathrm{e}$ mantidas neste estudo, o que permitiu observar de forma ampla os interesses e preferências de pesquisa de todos os pesquisadores da rede identificada.

\section{Etapa 4 - Identificação de colaboração acadêmica}

Após a seleção das pessoas que comporiam a rede de pesquisadores, utilizou-se o software scriptLattes ${ }^{19}$ a fim de compilar as informações pertinentes ao currículo de cada um.

Vale mencionar que os relatórios gerados pelo scriptLattes permitem avaliar, analisar e/ou documentar a produção de grupos de pesquisa, e/ou pesquisadores que se deseja conhecer. Adicionalmente, podem ser geradas redes de coautoria entre os membros do grupo de interesse, bem como um mapa de geolocalização dos mesmos. Embora o software gere relatórios de diferentes tipos de produções bibliográficas, para este estudo somente foram considerados a rede de coautoria e o mapa de geolocalização.

\section{Resultados}

Nesta seção são apresentados os resultados obtidos através das etapas descritas no método, que identificaram os descendentes acadêmicos dos pesquisadores precursores, ou seniores, previamente identificados, encontrando novos pesquisadores, descendentes ou 'filhos', ou seja, que tiveram relação acadêmica de orientação de mestrado, doutorado ou pós-doutorado com algum dos 61 pesquisadores precursores. Além disso, o estudo objetivou selecionar somente os pesquisadores que publicaram na área de interesse, a fim de identificar a rede de coautoria e comunicação científica estabelecida entre os membros.

Os resultados encontram-se organizados em tópicos, de acordo com as questões de pesquisa deste estudo.

\section{Identificação dos descendentes acadêmicos dos precursores com seleção dos pesquisadores que publicaram na área}

Foram identificados 143 pesquisadores, sendo 29 correspondentes aos pesquisadores precursores e 114 a seus descendentes (orientandos) que possuíam, ao menos, uma ocorrência das 78 combinações de termos, nos títulos de suas publicações presentes em seu currículo Lattes (artigos em periódicos; livros ou capítulos de livros; trabalho completo, resumo simples ou expandido em anais de eventos). Isso significa que foram relacionados somente aqueles que publicaram trabalhos na área de prevenção de drogas em contextos educacionais, tema deste estudo. Embora o número de orientações concluídas dos pesquisadores precursores seja de aproximadamente 1.743 orientandos, a maioria deles não apresentou publicações na 
área, indicando provavelmente sua atuação em outras áreas de pesquisa, correlatas a suas graduações e/ou programas de pós-graduação ou a possibilidade de estarem exercendo sua função no setor produtivo, após a titulação acadêmica.

Dos 114 pesquisadores descendentes, 8 eram estagiários de pós-doutorado, 60 orientandos de doutorado e 46 de mestrado. Apesar do grupo de precursores ser composto por 61 pessoas $^{18}$, somente 29 foram selecionados neste estudo, pois apresentavam publicações sobre o tema em questão em seus currículosv ${ }^{v}$.

Os resultados mostraram maior representatividade de mulheres pesquisadoras $(n=110)$ contrapondo ao número de homens pesquisadores $(n=33)$, equivalente a uma prevalência de $77 \%$ de mulheres.

\section{Ano de formação e localização geográfica}

Sobre a titulação dos membros da rede, constataram-se 113 doutores e 30 mestres, com as titulações adquiridas ao longo do tempo até o ano de 2017. Quanto ao ano da obtenção do título, entre os 113 doutores e 30 mestres, pouco mais da metade (55\%) se titularam nos últimos 10 anos, entre 2007 a 2017 (Figura 2).

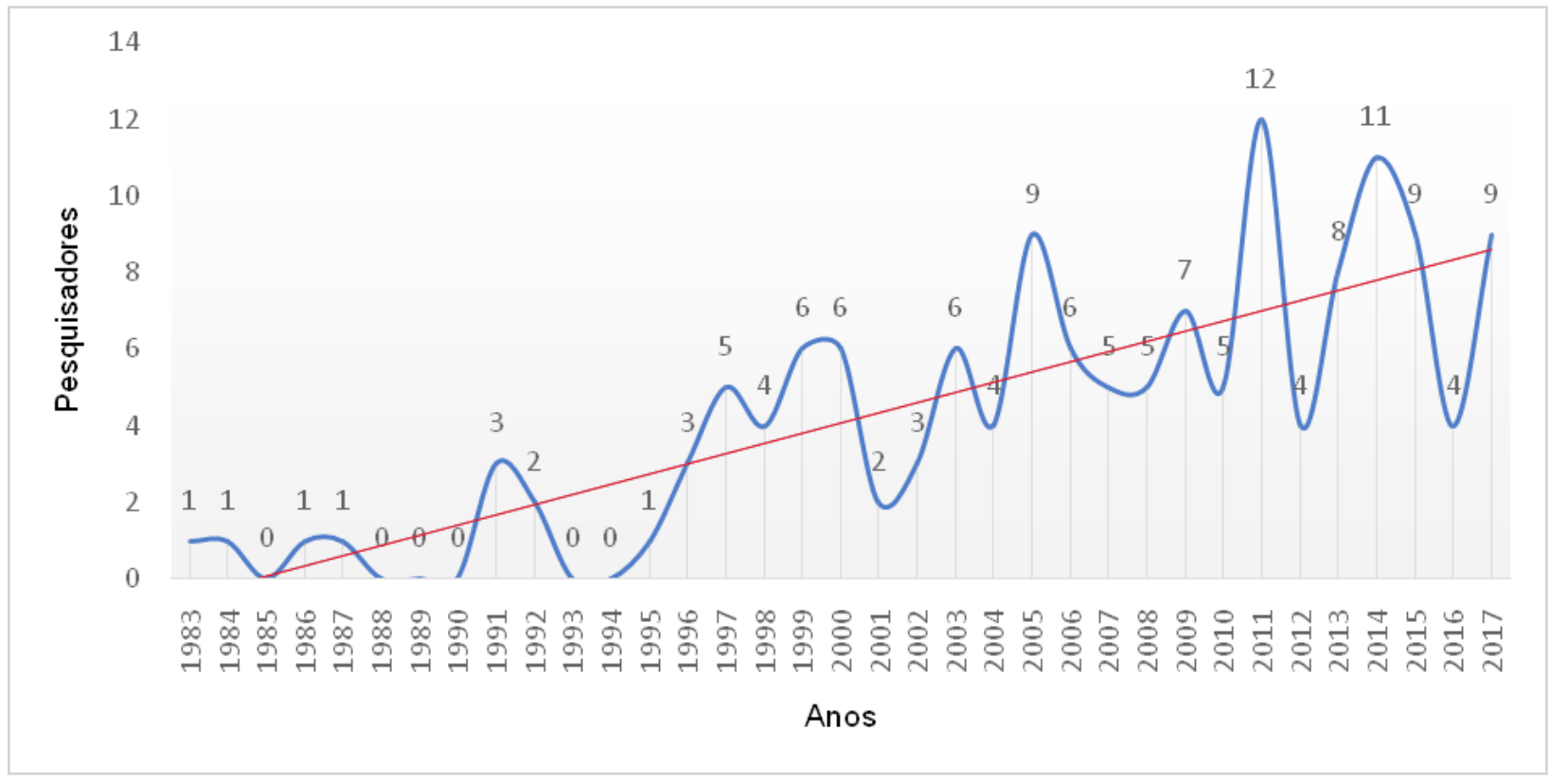

Figura 2 - Obtenção da titulação de mestrado ou doutorado por ano Fonte: Os autores (2018).

É possível verificar ainda que 2011 e 2014 foram os anos que mais formaram pessoas da rede, com 12 e 11 pesquisadores, respectivamente. Desde o ano de 1983, primeiro ano de titulação encontrado, os anos de 1985, 1988, 1989, 1990, 1993 e 1994 não tiveram nenhuma pessoa da rede com obtenção do título de mestre e/ou doutor.

Observa-se também um crescimento positivo de pesquisadores da área ao longo do tempo, independente dos anos com maior ou menor obtenção da titulação, demonstrado pela linha de tendência expressa na cor vermelha na Figura 2.

A Figura 3 mostra a localização geográfica da rede de pesquisadores dentro do território nacional, onde cada ponto verde representa um pesquisador. A localização corresponde à informação inserida pelo

v O estudo realizado para identificação dos precursores, considerou o Catálogo de teses e dissertações da Capes e identificou trabalhos sobre o tema investigado, por meio de resumos, títulos e palavras-chave, selecionando os orientadores de tais trabalhos que tiveram, ao menos, duas orientações no período considerado. Isto não significa que todos os orientadores tenham publicado na área os tipos de publicações elencados nos currículos Lattes e considerados neste estudo: artigo em periódico, capítulos e organizações de livros, resumos e trabalho completo em eventos. Assim, dos 61 pesquisadores precursores, somente 29 foram considerados neste estudo. 
pesquisador em seu currículo Lattes como endereço profissional. No presente estudo, dos 143 pesquisadores identificados, apenas 113 haviam inserido a informação em seu currículo. Conforme pode-se observar, a maioria dos pesquisadores que atua na área de prevenção de drogas no contexto educacional encontra-se na região Sudeste do país $(n=58)$, seguido pela região $S u l(n=24)$, região Nordeste $(n=18)$, região CentroOeste $(n=12)$ e região Norte $(n=1)$.

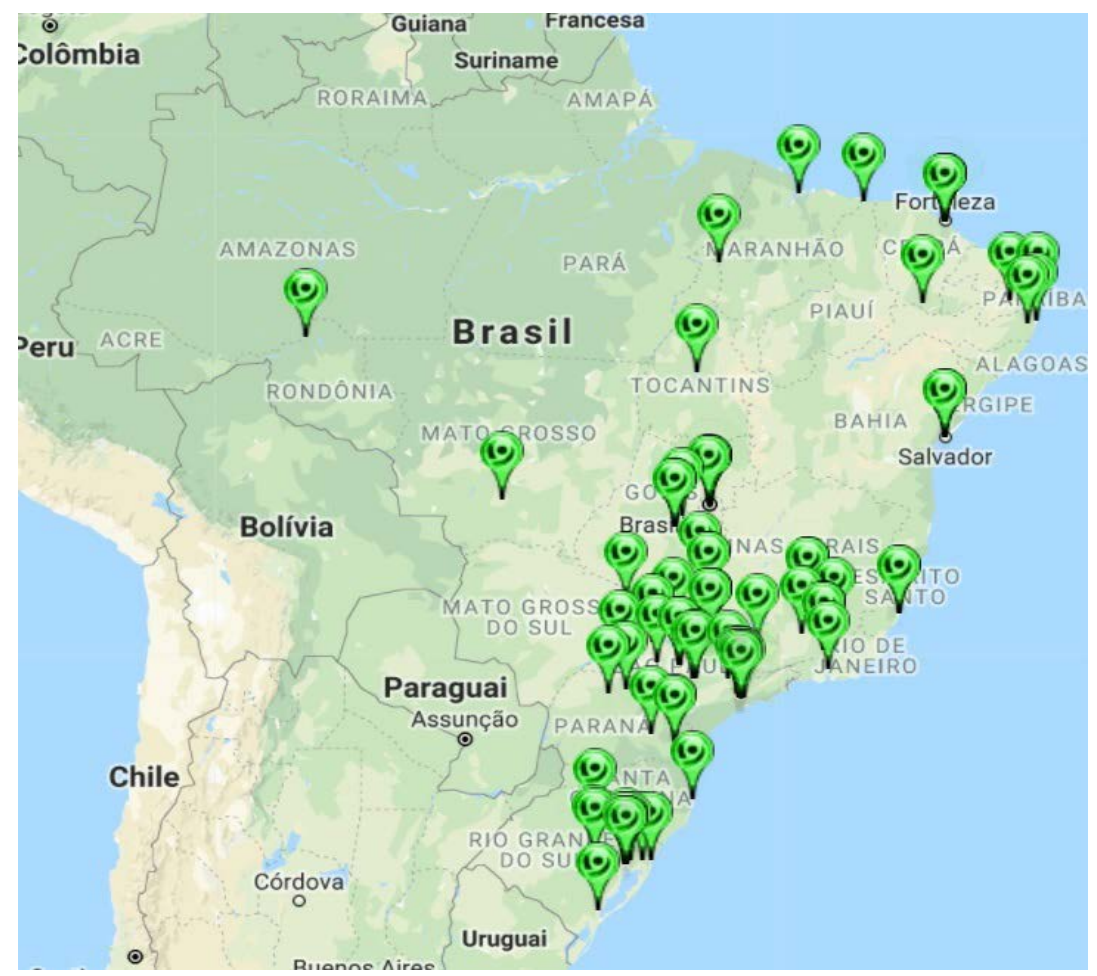

\begin{tabular}{|l|c|}
\hline Região & $\mathbf{N}^{\circ}$ \\
\hline Centro-Oeste & 12 \\
\hline Nordeste & 18 \\
\hline Norte & 1 \\
\hline Sudeste & 58 \\
\hline Sul & 24 \\
\hline Total geral & 113 \\
\hline
\end{tabular}

Figura 3 - Mapa de geolocalização da rede de pesquisadores Fonte: Os autores (2018).

O estado de São Paulo é o que aglutina maior quantidade de pesquisadores $(n=43)$, seguido do Rio Grande do Sul ( $n=19)$ e Minas Gerais ( $n=12)$. Juntos, esses estados somam 65\% dos pesquisadores da rede.

\section{Rede de coautoria}

A Figura 4 apresenta a rede de coautoria (grafos 'a' e 'b') entre os pesquisadores identificados, considerando as diferentes áreas de atuação. Cada ponto sinalizado nos grafos representa um pesquisador e as arestas representam a colaboração entre eles. Entende-se, para este fim, colaboração como publicação em conjunto, relações de coautoria de um ou mais trabalhos, considerando os diferentes veículos de comunicação científica: resumos e trabalhos completos em anais de eventos, livros e/ou capítulos e artigos em periódicos. A espessura de cada aresta na rede (Figura 4) é proporcional à quantidade de colaborações entre os pesquisadores interligados. Assim, arestas mais grossas caracterizam alto grau de colaboração enquanto as mais finas, menor grau. 


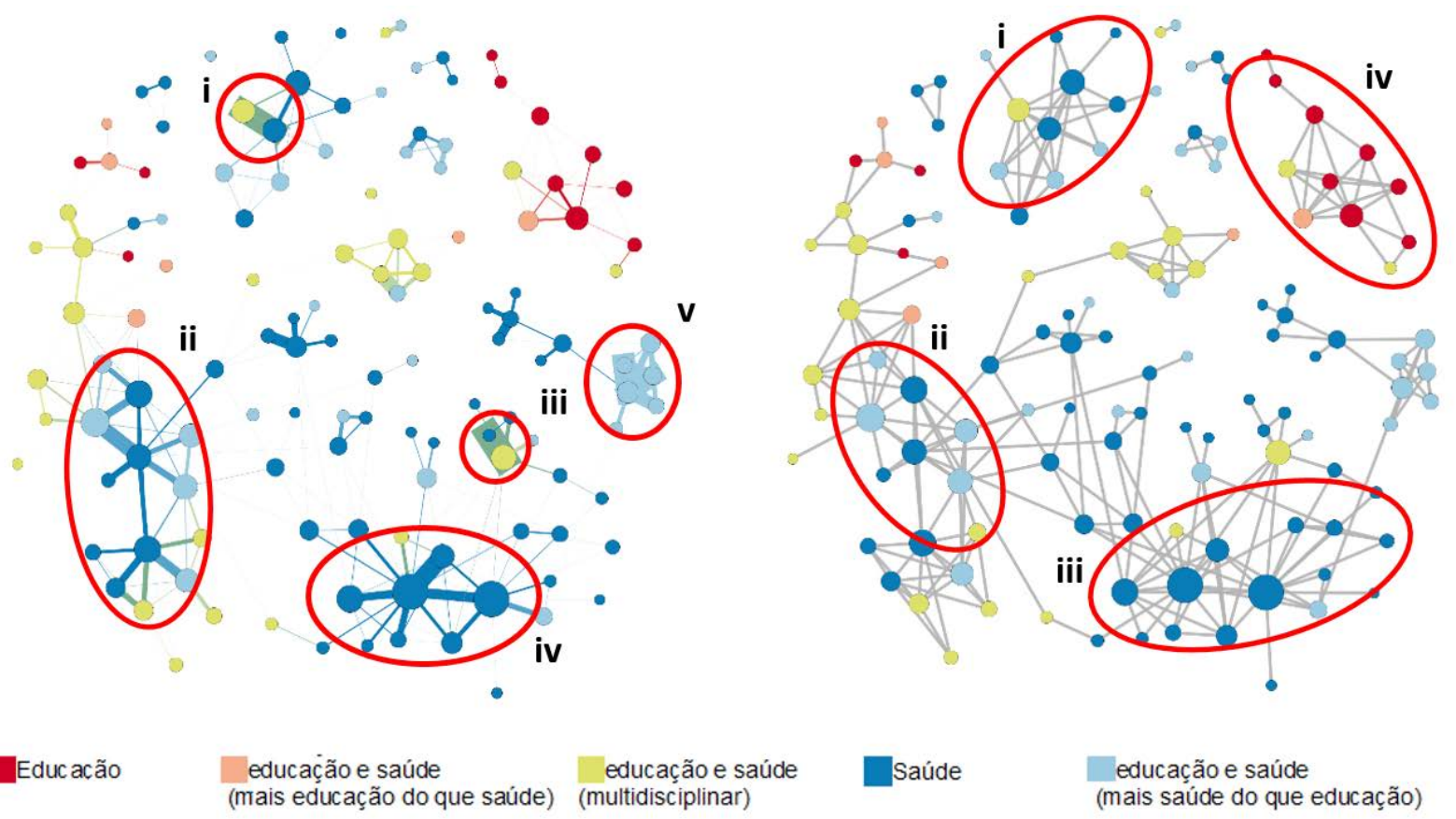

Figura 4 - Rede de coautoria entre pesquisadores: a) Rede com pesos; b) Rede sem pesos Fonte: Os autores (2018).

Seguindo o processo de classificação da etapa 3, descrita no método, a Tabela 1 apresenta as áreas de atuação dos pesquisadores e a quantidade de pesquisadores atuantes em cada respectiva área.

Tabela 1 - Pesquisadores por área de atuação

\begin{tabular}{l|c}
\multicolumn{1}{c|}{ Área } & Pesquisadores \\
\hline Educação & 13 \\
\hline Educação e saúde (mais educação do que saúde) & 7 \\
\hline Educação e saúde (mais saúde do que educação) & 32 \\
\hline Educação e saúde (multidisciplinar) & 27 \\
\hline Saúde & 64 \\
\hline Total & $\mathbf{1 4 3}$
\end{tabular}

Fonte: Os autores (2018).

Os pesquisadores representados na Figura 4 apresentaram ao menos uma colaboração em rede, porém foram encontrados 14 pesquisadores isolados, que não estão expressos na figura. Entre estes, 6 eram da área da saúde, 3 da área educação e saúde multidisciplinar, 2 da área educação e saúde (mais saúde), outros 2 da educação e 1 pesquisador da educação e saúde (mais educação). Este dado revela que 90\% dos pesquisadores colaboraram cientificamente com, ao menos, uma pessoa da rede.

\section{Rede de coautoria por área}

Verificou-se que a maior concentração de pesquisadores (45\%) que atuavam em temas relacionados à prevenção de drogas em contextos educacionais está na área de saúde $(n=64)$, sendo a área educação e saúde (mais educação do que saúde) e a área de educação as que apresentaram menor número de pesquisadores, $\mathrm{n}=7 \mathrm{e} \mathrm{n}=13$ respectivamente. 
Em relação à colaboração, nota-se maior interação entre os pesquisadores da área da saúde e estes com a área da educação e saúde em sua subárea - mais saúde do que educação e multidisciplinar, representados pelos pontos azul claro e amarelos, respectivamente (Figura 4). Os pontos vermelhos, que equivalem à área de educação, colaboraram entre si, porém não se ligavam diretamente a nenhum ponto relativo a pesquisadores da área da saúde; entretanto, apresentavam relação com as áreas educação e saúde multidisciplinar e mais educação do que saúde, Figura 4 (b) agrupamento iv. Além disso, dentre esses subgrupos, três apresentaram maior agrupamento de pesquisadores da saúde - em azul, Figura 4 (b) i, ii e iii. Um outro agrupamento evidencia os pesquisadores da educação - em vermelho, Figura 4 (b) iv.

Ainda na Figura 4, observa-se também que há subgrupos dentro da rede de coautoria que demonstravam relacionamentos mais fortes, representados por pontos e arestas mais grossas, agrupamentos que estão expressos na Figura 4 (a) identificados como i, ii, iii, iv e v. Tais relações mais intensas podem significar relações de orientação e/ou de parcerias entre pesquisadores de um mesmo instituto/departamento.

\section{Canais de comunicação científica e anos de publicação}

Os resultados apresentados na Tabela 2 se referem à comunicação científica, ou seja, às formas e canais utilizados pelos pesquisadore ${ }^{20} \mathrm{e}$ à disseminação de informações científicas entre os pares ${ }^{21}$, quantificadas em cada tipo.

Em relação à publicação dos pesquisadores, foram encontradas 154 publicações na área de prevenção de drogas em contextos educacionais, diluídas em trabalhos completos em anais de eventos, artigos publicados em periódicos, capítulos de livros e organização de livros (Tabela 2).

Tabela 2 - Tipo de publicação e quantidade

\begin{tabular}{lc} 
Tipo de publicação & Quantidade \\
\hline Trabalho completo em anais de evento & 32 \\
\hline Artigo em periódico & 43 \\
\hline Capítulo de livro & 52 \\
\hline Livro & 27 \\
\hline Total & $\mathbf{1 5 4}$
\end{tabular}

Fonte: Dados da pesquisa (2018).

Foram retirados desta contagem os resumos, pois acredita-se que os mesmos possuam menor impacto na comunicação científica e não representem estudos e projetos na íntegra.

As publicações foram identificadas nos títulos correspondentes a cada uma, no currículo Lattes dos pesquisadores, por meio das 78 combinações de termos, previamente definidos e validados, como estratégia de busca. Esta estratégia possibilitou selecionar tanto os currículos relevantes, portanto cada um dos pesquisadores componentes da rede, quanto as publicações dentro de cada currículo.

O número de publicações foi positiva em relação aos anos, ou seja, foi crescente dentro da área de interesse nos últimos 30 anos (Figura 5). O período de 2010 até 2017 condensa a maioria das publicações, aproximadamente $55 \%$ de toda a produção ( 85 trabalhos), seguido por 58 publicações entre os anos de 2000 e 2009. Até o ano de 1989 foram identificados somente dois trabalhos publicados, sendo um capitulo de livro e um artigo em periódico e, na década compreendida entre 1990 e 1999, apenas 9 publicações. 


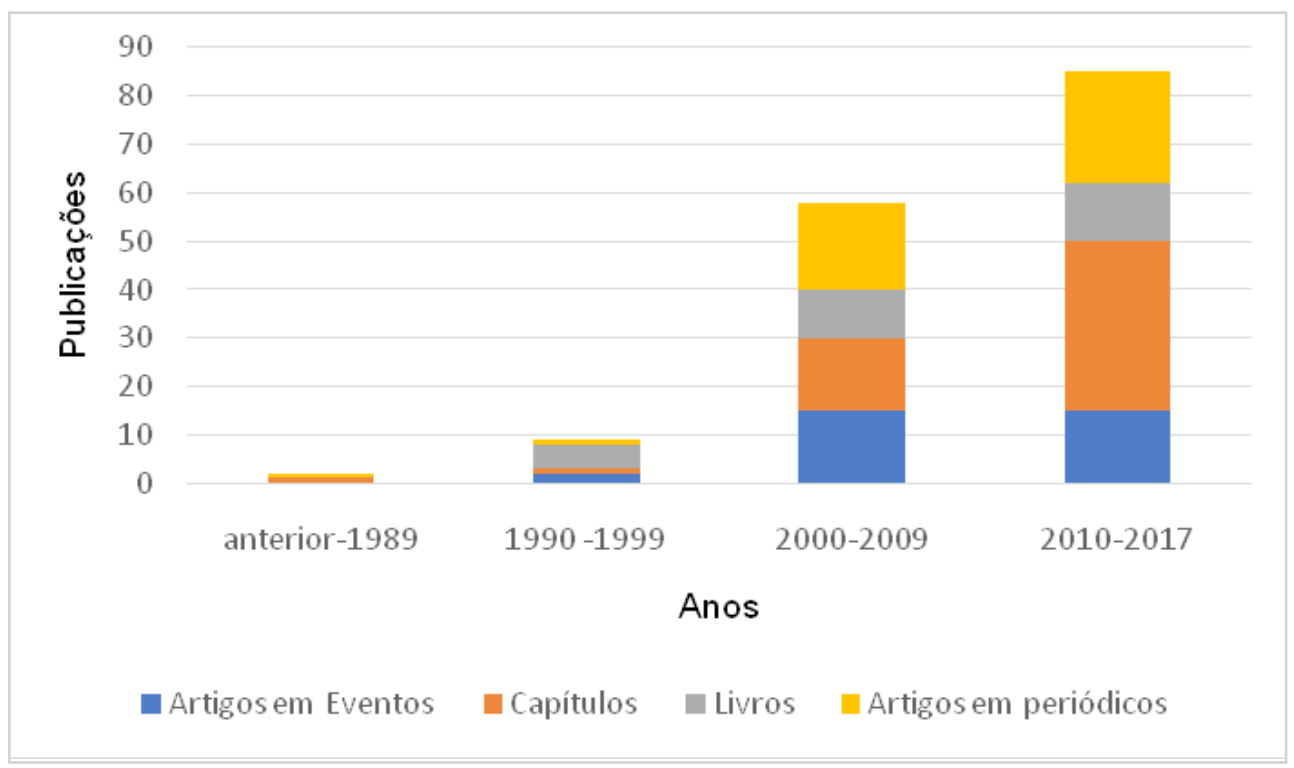

Figura 5 - Publicações classificadas por tipo e ano Fonte: Os autores (2018).

Sobre as categorias e os veículos de publicação, constatou-se que o canal de comunicação científica mais utilizado pelos pesquisadores são os eventos científicos, através da de resumos (simples e expandidos) e trabalhos completos publicados em anais de eventos. As editoras e os periódicos somaram resultados muito próximos, com 30 editoras diferentes entre os livros e capítulos, retirando as duplicatas, e os periódicos 32 diferentes (Tabela 3).

Tabela 3 - Quantidade de publicações por tipo e veículos diferentes

\begin{tabular}{l|l|c|c}
\multicolumn{2}{l|}{} & $\begin{array}{c}\text { No de } \\
\text { trabalhos }\end{array}$ & $\begin{array}{c}\text { Veículos } \\
\text { diferentes }\end{array}$ \\
\hline \multirow{3}{*}{ Eventos científicos } & Resumos simples & 133 & 102 \\
\cline { 2 - 4 } & Resumos expandidos & 10 & 10 \\
\cline { 2 - 4 } & Trabalhos completos & 32 & 29 \\
\hline \multirow{2}{*}{ Editoras } & Livros & 27 & 18 \\
\cline { 2 - 4 } & Capítulos de livros & 52 & 24 \\
\hline Periódicos & Artigos & 43 & 32 \\
\hline
\end{tabular}

Fonte: Os autores (2018).

Verificou-se grande dispersão na quantidade de publicações nos diferentes veículos de cada categoria entre eventos, editoras e periódicos. Ou seja, não foi possível identificar os veículos de publicação mais frequentes como os principais eventos, as editoras e os periódicos que mais divulgam trabalhos sobre prevenção e drogas em contextos educacionais.

Considerando que o tema em estudo transita em áreas multidisciplinares, realizou-se uma análise da estratificação de cada um dos 32 periódicos identificados, bem como seus respectivos fatores de impacto, com vistas a verificar a presença de tendência de publicação em áreas específicas. Para tal análise, foi realizada uma busca dos estratos de cada um dos 32 periódicos na plataforma Sucupira (Capes), através da avaliação Qualis, nas áreas de ensino, educação, saúde coletiva, enfermagem e psicologia. O resultado demonstrou que a representatividade é similar entre elas e que a publicação do tema acontece em revistas 
com impacto em várias áreas. Portanto, a hipótese de possível tendência para publicação em determinada área ou determinado periódico foi descartada.

Os principais resultados referentes às relações de coautoria, localização, produção intelectual e divulgação científica da rede de pesquisadores da área de prevenção de drogas em contextos educacionais, foram evidenciados, de acordo com os critérios definidos e desenho metodológico adotados.

\section{Discussão}

Considerando os resultados encontrados, observou-se que, embora a identificação de todos os descendentes acadêmicos dos precursores tenha gerado um número grande de pessoas, apenas um grupo reduzido tem produzido e difundido estudos na área de prevenção de drogas em contextos educacionais. Entretanto, a formação de aproximadamente 1.743 pessoas ao longo do tempo (total de pessoas encontradas com a identificação dos descendentes dos precursores) é um número considerado alto, pensando nos 61 precursores e seus filhos acadêmicos 'diretos' em uma rede nova, com formação de mestres e doutores recentes.

Analisar a herança intelectual perpetuada através das relações de orientação é proporcionar um conhecimento quantitativo da capilaridade e alcance da ciência perpassado em gerações, chamado de genealogia acadêmica, que visa analisar e documentar essas ligações. Um estudo de genealogia acadêmica realizado sobre o pesquisador César Lattes, encontrou 425 sucessores até o ano de 2017, dos quais: 7 filhos acadêmicos diretos, 75 netos, 193 bisnetos, 148 trinetos e dois tetranetos ${ }^{22}$. Considerando o referido dado, relativo a um pesquisador da década de 1940 e seus descendentes diretos e indiretos em relações acadêmicas ainda crescentes, é possível verificar o potencial de uma relação de orientação.

A possibilidade de um orientador formar estudantes dentro dos programas de pós-graduação e propagar conhecimento científico influi diretamente na qualidade das instituições de ensino e em sua capacidade de promover o desenvolvimento tecnológico e socioeconômico do país ${ }^{23}$.

Em relação aos resultados verificados no presente estudo, não é de se surpreender que um número menor a todas as orientações dos precursores, as quais somaram 1.743 - entre seus alunos de mestrado, doutorado e supervisões de pós-doutorado - se refere a pesquisadores atuantes na área em questão. Como já mencionado, a área de conhecimento sobre prevenção de drogas em contextos educacionais se caracteriza pela multidisciplinaridade e interesse específico daqueles que se propõem a realizar pesquisa sobre o tema e, nesse sentido, obteve-se um número reduzido de 114 descendentes, o que corresponde a $6,5 \%$ de todos os filhos diretos dos precursores.

O resultado observado sobre o crescimento ascendente no número de pesquisadores - mestres e doutores -, formados ao longo dos anos (Figura 2), corrobora os dados de investimentos em pós-graduação no Brasil. Segundo Ramos e Velho ${ }^{24}$, na década de 1970 havia aproximadamente 800 cursos de mestrado e doutorado e, em 2008, a quantidade era da ordem de 3.700 cursos. Artes e Mena-Chalco ${ }^{25}$ conduziram um mapeamento dos estudos realizados sobre relações raciais em cursos brasileiros de pós-graduação e também verificaram um aumento progressivo no número de alunos ao longo dos anos, que defenderam estudos sobre o tema. Além disso, nossos resultados estão alinhados às evidências encontradas pelos autores ${ }^{25}$ e pelos dados do IBGE coletados em âmbito nacional ${ }^{26}$ que indicam uma porcentagem maior de mulheres pós-graduandas comparada à de homens.

A maior concentração de pesquisadores nas regiões Sudeste e Sul do país demonstra a distribuição desigual de Instituições de Ensino Superior (IES) no território nacional, com uma concentração de 48\% das IES no Sudeste, evidenciada no censo sobre educação superior de $2013^{26}$. Similar evidência foi discutida no trabalho de Bernardo e colaboradores ${ }^{18}$, em relação ao grupo de precursores e observado em outros estudos $^{15,25}$. Esta questão se estende aos programas de pós-graduação. Embora o Plano Nacional de PósGraduação, desde a versão de 2005-2010, apresente propostas para minimizar essas desigualdades, elas 
ainda existem. Segundo Santos e Azevedo ${ }^{27}$, em 2008, 61,1\% dos programas de mestrado e doutorado eram oferecidos na região Sudeste.

Pesquisas sobre trabalhos colaborativos, redes de pesquisadores e suas produções científicas podem ser um norteador sobre um tema de interesse, o que requer a escolha do método e instrumentos adequados. A ferramenta scriptLattes já foi utilizada para análise de diversas redes de colaboração entre pesquisadores atuantes no Brasil ${ }^{12,15,28}$, fornecendo dados sobre a produção científica e as relações acadêmicas de determinada área ou campo do conhecimento.

Os dados deste estudo demonstraram uma relação de colaboração entre a maioria dos pesquisadores e, embora não tenham sido realizadas análises utilizando-se índices bibliométricos, percebe-se a existência de pequenos subgrupos, dentro da rede maior, o que pode significar uma relação de orientação, ou uma ligação de departamentos e universidades específicas. Entretanto, embora a rede identificada seja pequena, com 143 pesquisadores (do universo 1.804 composto de orientadores e seus orientados), os resultados evidenciaram o distanciamento entre pesquisadores das áreas da saúde e da educação, quanto a práticas de coautoria, ou colaborativas.

Em relação à consistência e densidade das redes de colaboração de áreas especificas do conhecimento, Mena-Chalco e colaboradores ${ }^{28}$ realizaram um estudo contendo uma análise ampla sobre a colaboração entre os pesquisadores que possuem currículo Lattes das oito grandes áreas do conhecimento estabelecidas pela Capes, a saber: ciências exatas e da terra, ciências biológicas, engenharias, ciências da saúde, ciências agrárias, ciências sociais aplicadas, ciências humanas e a área de linguística, letras e artes. O referido estudo identificou diversos indicadores sobre as redes compostas. Um dado que merece ser mencionado é que a área das ciências humanas, na qual se encontra a educação, é menos densa em relação a suas arestas, ou colaborações, quando comparada com a das ciências da saúde.

No mesmo sentido, Leite e colaboradores ${ }^{29}$ utilizaram-se da teoria de redes para desenvolver uma metodologia de análise de currículos de pesquisadores, com uso de programas computacionais para construção de planilhas de dados, contagem de coautorias e análise de redes sociais. Os sujeitos investigados eram pesquisadores $1 \mathrm{~A}$ do $\mathrm{CNPq}$, líderes de grupo de pesquisa nas áreas de educação, engenharia de produção e física. Um resultado que chamou a atenção refere-se ao comportamento diferencial dos pesquisadores da área da educação. Segundo os autores, na educação, o pesquisador principal (que representa o ponto central de um nó da rede, chamado de 'ego') publicou solitariamente 57,4\%, com mais um membro da rede 25,3\% e, com menos frequência, equivalente a 11,5\% com três autores; já nas outras áreas avaliadas a publicação em colaboração teve maior prevalência ${ }^{14}$. Outro estudo específico com pesquisadores da área de educação realizado por meio de entrevistas, mostrou que, no geral, eles acreditam numa concepção de trabalho em rede diferente daquela levada em conta pelos pesquisadores de outras áreas, e com crença em certos pressupostos que podem limitar a comunicação científica e o trabalho colaborativo. A crítica principal feita pela autora está no sentido de a colaboração científica ser um componente central dos modos de se fazer ciência na contemporaneidade, o que não tem sido muito considerado por pesquisadores da educação ${ }^{29}$.

Os resultados encontrados neste estudo se alinham aos das pesquisas mencionadas, no sentido da observação de uma rede de educação que não tem relação de colaboração com a saúde, como um padrão que tem sido identificado em outros estudos. Longe de esgotar a questão, a proposta é que esta discussão sirva de incentivo para que outros estudos sejam realizados para um melhor aprofundamento sobre o assunto. Vale mencionar que esse fenômeno extravasa este estudo, especialmente em relação ao trabalho colaborativo e à intersetorialidade, que ultrapassa o âmbito acadêmico, estendendo-se a outros contextos ou campos de atuação. Segundo Mendes e Akerman ${ }^{30}$ "A prática da intersetorialidade aponta que não há receitas, metodologias consagradas ou evidências estabelecidas. Há, sim, algumas experiências, tentativas, erros e acertos". Assim, a intersetorialidade pode ser entendida como o grau de comunicação entre os setores sem ignorar os saberes específicos de cada área, por meio da responsabilidade compartilhada para enfrentar e superar determinadas situações, de forma que os objetivos sejam alinhados ${ }^{31}$. 
A realização de estudos sobre prevenção de drogas em contextos educacionais requer esforços de várias áreas, visando à transformação da lógica social e à formulação de proposições de resolubilidade dos problemas encontrados na sociedade atual, tendo em vista que não é uma questão só da saúde ou só da educação, mas de muitos outros atores sociais.

Em relação às publicações sobre o tema investigado no presente estudo, todos os tipos identificados apresentam tendência crescente de produção no período analisado, ainda que, ocasionalmente, mostrem períodos de baixa produção. Quanto ao número de publicações, os resumos foram a forma mais comum de comunicação científica utilizada pelos pesquisadores da rede, seja pela facilidade de divulgação seja pela facilidade de execução desse tipo de trabalho.

Os artigos científicos em periódicos, por sua vez, são a forma de comunicação cuja função primária é divulgar resultados de pesquisas de forma rápida e concisa; além disso, estabelecem uma velocidade de divulgação e intercâmbio entre pesquisadores, promovendo novas pesquisas e interesses e aumentando o volume de produção sobre determinado assunto ${ }^{15}$. Neste sentido, no presente estudo, foram identificados 43 artigos sobre prevenção de drogas no contexto educacional, publicados até o ano 2017, sendo a sua maior concentração nos anos de 2010 a 2017. Embora o número pareça pequeno, outras pesquisas bibliométricas de áreas interdisciplinares ou multidisciplinares, revelaram dados parecidos.

Um estudo sobre saúde mental e educação, realizado por meio de análise bibliométrica, identificou 43 artigos publicados entre os anos de 1968 e 2014. Os resultados indicaram que a última década reúne a maior parte dos estudos, e as normativas de caráter internacional no campo da educação e da saúde mental podem ter estimulado esse interesse crescente em pesquisas sobre o tema nos últimos anos ${ }^{31}$.

Em um outro estudo da área multidisciplinar sobre adolescentes em conflito com a lei, foram identificados 42 artigos distribuídos em diferentes bases de dados. Os autores concluíram que há uma precária assistência e promoção da saúde do adolescente privado de liberdade, sendo tais desafios complexos e multifatoriais, seja pela dificuldade de articulação da rede de saúde no atendimento ao adolescente institucionalizado, seja pela persistência da lógica punitiva nos estabelecimentos destinados à socioeducação ${ }^{32}$.

Não obstante, parece que os investimentos em ciência e tecnologia (C\&T) e comunicação científica vêm crescendo, conforme aponta o estudo de Costa e Leite ${ }^{33}$. Os autores coletaram e analisaram dados sobre investimento/financiamento em C\&T, advindos de várias fontes de informação. A partir dos resultados encontrados, é possível indicar que Brasil, Argentina e México concentram maior parte do investimento em C\&T e que o crescimento das publicações latino-americanas se deve, em grande parte, ao crescimento das publicações científicas no Brasil. Outro dado verificado indica que a região tem apresentado consideráveis avanços nos últimos dez anos, tanto em investimento em pesquisa como no desenvolvimento de mecanismos para a comunicação e divulgação da ciência ${ }^{33}$.

Tomados em conjunto, espera-se que o volume de publicações e colaborações entre pesquisadores possa sanar algumas questões e problemas sociais eminentes. Não há dúvida de que o aumento de publicações e divulgação sobre a prevenção de drogas em contextos educacionais favorecerá uma maior e melhor articulação entre pesquisadores que atuam na área, resultando num melhor delineamento das estratégicas práticas até então adotadas, bem como incentivando o compartilhamento dos conhecimentos e das experiências entre grupos.

\section{Considerações finais}

O mapeamento e a identificação dos pesquisadores da área em questão revelou a existência de uma rede colaborativa, localizada em grande parte na região Sudeste do país. A análise da rede demonstrou maior colaboração entre pesquisadores da saúde, e nenhum relacionamento direto entre pesquisadores da área da educação com pesquisadores da área da saúde, sendo este comportamento também observado em outros estudos como apontado na seção Discussão. 
A área de prevenção de drogas em contextos educacionais é um tema recente em termos de pesquisas no Brasil e que necessita de maiores investimentos e integralidades de informações entre os campos do conhecimento envolvidos, para que a ciência produzida em âmbito acadêmico culmine em ações práticas mais eficazes.

Dessa forma, a relevância do presente estudo centra-se na apresentação de uma rede, até então desconhecida, de pesquisadores precursores e seus descendentes acadêmicos que atuam na temática relativa à prevenção de drogas no contexto educacional. Tal identificação favorecerá a articulação entre os membros da rede, bem como o estabelecimento de práticas colaborativas entre os pesquisadores sobre o tema, dirimindo a realização de estudos e práticas isoladas e promovendo o avanço da ciência e das políticas públicas condizentes com a prevenção.

Como desdobramento natural deste trabalho podemos mencionar a análise qualitativa dos estudos realizados pela rede de pesquisadores identificada, sobre os temas relacionados à prevenção de drogas em contextos educacionais. Acreditamos que tal análise poderá trazer novas contribuições e informações relevantes sobre a ciência produzida no país acerca do tema e, assim, promover uma reflexão de como tal ciência dialoga com a prática de prevenção.

\section{Referências}

1. Sodelli M. Uso de drogas e prevenção: da desconstrução da postura proibicionista às ações redutoras de vulnerabilidade. São Paulo: Iglu; 2010.

2. Souza FB, Andrade ALM, Rodrigues TP, Nascimento MO, De Micheli D. Avaliação das concepções de educadores de escolas públicas e particulares sobre uso de drogas: um estudo exploratório. Est. Pesq. Psicol. 2015;15(3):1081-1095.

3. Moreira A, Vóvio CL, De Micheli D. Drug abuse prevention in school: challenges and possibilities for the role of the educator. R. Educ. Pesq. São Paulo. 2015;41(1):119-134.

4. Foxcroft DR, Tsertsvadze A. Universal alcohol misuse prevention programmes for children and adolescents: Cochrane systematic reviews. Perspect Public Health. 2012;132(3):128-134.

5. Strøm HK, Adolfsen F, Fossum S, Kaiser S, Martinussen M. Effectiveness of school-based preventive interventions on adolescent alcohol use: a meta-analysis of randomized controlled trials. Subst Abuse Treat Prev Policy. 2014;9:48.

6. Nascimento MO, De Micheli D. Evaluation of different school-based preventive interventions for reducing the use of psychotropic substances among students: a randomized study. Ci. Saúde Coletiva. 2015; 20(8):2499-2510.

7. Babor TF, Caetano R, Casswell S, Edwards G, Giesbrecht N, Graham K. Alcohol: no ordinary commodity: research and public policy. 2 ed. New York: Oxford University Press; 2010.

8. Cahill HW. Challenges in adopting avidence-based school drug education programmes. Drug Alcohol Rev. 2007;26(6):673-9.

9. United Nations Office for Drug Control and Crime. Prevention Lessons learned in drug abuse prevention: a global review. Nova Iorque; 2002.

10. Hanley SM, Ringwalt C, Ennett ST, Vincus AA, Bowling JM, Haws SW, Rohrbach LA. The prevalence of evidence-based substance use prevention curricula in the nation's elementary schools. J Drug Educ. 2010;40(1):51-60.

11. World Health Organization (WHO). School health and youth health promotion: facts [Internet]; 2011 [cited 2019 jul 18]. Disponível em: http://www.who.int/school_youth health/facts/en/index.html

12. Ferraz RRN, Quoniam L, Alvares LMAR. Avaliação de redes multidisciplinares com a ferramenta scriptLattes: os casos da nanotecnologia, da dengue e de um programa de pós-graduação Stricto Sensu em Administração. R. Eletr. Bibl. Ci. Inf. 2014;19(40):67-98.

13. Sobral NV, Silva FM, Bufrem LS, Coêlho MRCD. Produção científica colaborativa na área da saúde tropical: uma análise da rede de colaboração do Programa de Pós-Graduação em Medicina Tropical da Universidade Federal de Pernambuco. R. Eletr. Comun. Inf. Inov. Saúde. 2016 jan.-mar; 10(1):1-15. 
14. Leite D, Caregnato CE, Lima EGS, Pinho I, Miorando BS, Da Silveira PB. Avaliação de Redes de Pesquisa e Colaboração. Avaliação (Campinas; Sorocaba) SP. 2014;19(1):291-312.

15. Danuello JC, De Oliveira EFT. Análise cientométrica: produção científica e redes colaborativas a partir das publicações dos docentes dos programas de pós-graduação em Fonoaudiologia no Brasil. Em Questão. 2012;18:65-79.

16. Gatti BA. Formação de grupos e redes de intercâmbio em pesquisa educacional: dialogia e qualidade. R. Bras. Educ. 2005;30:124-181.

17. Price, D, Gursey S. Studies in scientometrics. Part 1. Transience and continuance in scientific authorship. In: International Forum on Information and Documentation. 1976; 17-24.

18. Bernardo JF, Pinheiro BO, Mena-Chalco JP, De Micheli D. Mapeamento de pesquisadores precursores da área de prevenção de drogas em contextos educacionais no Brasil. R. Eletr. Comun. Inf. Inov. Saúde. 2018 abr-jun.;12(2):172-88.

19. Mena-Chalco JP, CesarJunior RM. ScriptLattes: An open-source knowledge extraction system from the Lattes platform. J. Braz. Comp. Soc. 2009;15(4):31-9.

20. Velho L. A ciência e seu público. Transinformação [Internet]. 1997 [citado em 2019 jul 19]; 9(3):5-32. Disponível em: http://periodicos.puccampinas.edu.br/seer/index.php/transinfo/article/ viewFile/1575/1547

21. Bueno, WC. Comunicação cientifica e divulgação científica: aproximações e rupturas conceituais. Inf Inf. 2010;15(1):1-12.

22. Damaceno RJP, Mena-Chalco JP. O rastro acadêmico do professor César Lattes. In: I Workshop @ NUVEM; 2017 nov 21-22; Santo André, Brasil: UFABC; 2017.

23. Fava-de-Moraes F. Universidade, inovação e impacto socioeconômico. São Paulo Perspec. 2000;14(3):8-11.

24. Ramos MY, Velho L. Formação de doutores no Brasil e no exterior: impactos na propensão a migrar. Educ Soc. Campinas. 2011;32(117): 933-51.

25. Artes A, Mena-Chalco JP. Expansão da temática relações raciais no banco de dados de teses e dissertações da Capes. Educ. Pesq. 2017;43(4):1221-38.

26. Instituto Nacional de Estudos e Pesquisas Educacionais Anísio Teixeira (INEP). Censo da educação superior 2013: resumo técnico [Internet]. Brasília; 2015 [citado em 2019 jul 19]. Disponível em: http:// inep.gov.br/inep-data.

27. Santos AL, Azevedo JML. A pós-graduação no Brasil, a pesquisa em educação e os estudos sobre a política educacional: os contornos da constituição de um campo acadêmico. R. Bras. Educ. Rio de Janeiro. 2009;14(42):534-605.

28. Mena-Chalco JP, Digiampietri LA, Lopes FM, Cesar Junior RM. Brazilian bibliometric coauthorship networks. J. Assoc. Inf. Sci. Tech. 2014;65(7):1424-45.

29. Leite D. Conhecimento em educação: um olhar desde o estudo sobre redes de pesquisa e colaboração ou os sapatos da educação. Avaliação (Campinas). 2014;19(3):773-88.

30. Mendes R., Akerman M. Intersetorialidade: reflexões e práticas. In: Fernandez, JCA, Mendes R., organizadores. Promoção da saúde e gestão local. São Paulo: Hucitec; Cepedoc, 2007.

31. Tano BL, Hayashi MCPI. Saúde mental infantojuvenil e educação: análise bibliométrica da produção científica nacional e internacional. R. Eletr. Comun. Inf. Inov. Saúde. 2015; 9(3): 1-26.

32. Arêas Neto NTA, Constantino P, Assis SG. Análise bibliográfica da produção em saúde sobre adolescentes cumprindo medidas socioeducativas de privação de liberdade. Physis. Rio de Janeiro. 2017;27(3):511-540.

33. Costa MP, Leite FCL. Descrição preliminar do cenário da comunicação científica na América Latina e Caribe. R. Eletr. Comun. Inf. Inov. Saúde. 2017;11(2):1-15. 